\title{
Forced Convection Heat Transfer in V-Pattern Folded Core Sandwich Structures
}

\author{
Sifeng Li $(\mathbb{D}$, Zhijin Wang $\mathbb{D}$, and Chen Zhou \\ Minister Key Discipline Laboratory of Advanced Design Technology of Aircraft, Nanjing University of Aeronautics and Astronautics, \\ Nanjing 210016, China \\ Correspondence should be addressed to Zhijin Wang; zhijin@nuaa.edu.cn
}

Received 19 October 2021; Revised 19 January 2022; Accepted 26 January 2022; Published 10 February 2022

Academic Editor: Juan Du

Copyright (c) 2022 Sifeng Li et al. This is an open access article distributed under the Creative Commons Attribution License, which permits unrestricted use, distribution, and reproduction in any medium, provided the original work is properly cited.

Fully developed laminar flow and heat transfer performance of V-pattern folded core sandwich structures subjected to forced convection are numerically investigated. Based on the periodic feature of folded core, a methodology to simulate the fully developed flow and heat transfer state with a few unit cells is developed. The influences of geometric parameters on the coolant flow resistance and heat transfer behaviour are evaluated. Afterwards, approximate models are established based on design to optimize the heat dissipation efficiency. Design variables considered are geometric parameters of the V-pattern folded core, while the corresponding relative density is taken as a constraint. The simulation results show that the presented methodology makes it possible to analyse the fully developed regime with less computing resources. It is found that both pressure drop and heat transfer rate increase with the tortuosity of the flow channel and the inclination of the folded core. The optimization generates a design with a mildly tortuous feature in the streamwise direction to gain a maximum of heat dissipation efficiency under the current conditions.

\section{Introduction}

Sandwich structures are widely used in aerospace industry due to their lightweight, high specific strength, and high specific stiffness characteristics [1]. A folded core sandwich structure features various geometric parameters together with its open cell geometry, which gives it the potential for simultaneous load bearing and heat dissipation $[2,3]$. It consists of two face sheets and a three-dimensional core which can be folded from a planar base material along certain geometric lines $[4,5]$. The folded core can be manufactured from a variety of sheet materials and can be tailored into different geometries meeting different functional requirements. In addition, the open cell design of folded core allows internal fluid transport, further improving the heat dissipation capacity [3]. The mechanical and impact behaviours of V-pattern folded core sandwich structure have been paid much more attention and studied both experimentally and numerically in recent years, while little work exists on its active cooling capabilities [3, 5-7].
The fluid flow and heat transfer characteristics of sandwich structures with honeycomb, prismatic, and lattice cores have been extensively addressed in recent years. Wen et al. $[8,9]$ investigated the effects of topology on convective heat dissipation within metallic cellular structures both experimentally and numerically; also, an optimization study based on analytical results was performed. The results showed that cellular sandwich heat sinks having triangular cells were superior to square cells, both thermally and mechanically. Kim et al. [10] carried out experimental and numerical studies for lattice-frame materials to identify the local fluid flow features and their influence on the local and overall pressure and heat transfer characteristics. It was found that the horseshoe vortices were formed in front of the vertices of the LFM and the arch-shaped vortices were formed behind the vertices. The delay of the flow separation might lead to an increase in the overall heat transfer. Gao and Sun $[11,12]$ studied the thermal control of lattice truss core sandwich structures subjected to an internal thermal load under forced water convection. The results suggested that with an increase 
of inclination angle of truss, the heat transfer efficiency was improved, leading to a reduction of structural maximum temperature. Rakow and Waas [13] conducted a series of numerical and experimental studies to investigate the heat transfer and structural response of actively cooled metal foam sandwich panels. Feng et al. [14] numerically investigated the heat transfer performance of finned metal foam heat sinks subjected to uniform impinging flow and quantified the effects of all relevant configurational parameters of the heat sink upon the thermal performance. It was demonstrated that the finned metal foam heat sinks outperformed the plate-fin heat sinks on the basis of given weight or given pumping power. Shenoy et al. [15] also introduced forced convective flow and heat transfer characteristics in a wavy horizontal channel. Rashidi et al. [16, 17] studied the condensation flow inside rough and smooth nanochannels by attaching the roughness on the lower walls and investigated the effect of rough surface on the interface properties of the condensation flow for different roughness shapes. Results indicated that the condensation process of flow can be empowered by enhancement of the roughness height. Khoshvaght-Aliabadi et al. [18-21] performed experimental studies for forced convective flows of different nanofluids through a corrugated wavy channel and discussed the effects of different geometrical parameters and composition of fluid mixtures. Subsequently, laminar convection through the straight minichannel and wavy minichannel with various cross-section geometries was studied numerically, and the results depicted higher values of the heat transfer rate and pumping power for the wavy minichannel compared to the straight minichannel. However, as a promising candidate for multifunctional design, studies on the heat transfer performance of the folded core are still limited, which makes it necessary and indispensable to investigate their heat dissipation capacity.

The present study establishes an approach to simulate the fully developed heat transfer problem of $\mathrm{V}$-pattern folded core sandwich structures. Both friction factor and Nusselt number are evaluated using the method of computational fluid dynamics (CFD). The effects of the folded core geometric parameters on the pressure and heat transfer characteristics are investigated to gain an initial insight into the problem. Finally, the heat dissipation efficiency is optimized against relative density followed by a short discussion on the optimal results.

\section{Numerical Model}

2.1. V-Pattern Folded Core Sandwich Structures. A typical sandwich structure with V-pattern folded cores is shown in Figure 1. The top-face sheet is subjected to a uniform heat flux while the bottom-face sheet is assumed to be adiabatic. Forced convection is performed in channels (along the $X$ direction) formed by the $\mathrm{V}$-pattern folded cores and face sheets, which means that most heat is dissipated and taken away. Specifically, two different types of channels are identified in the structure, i.e., the channel with the triangular cross-section and the channel with the inverted triangular cross-section.

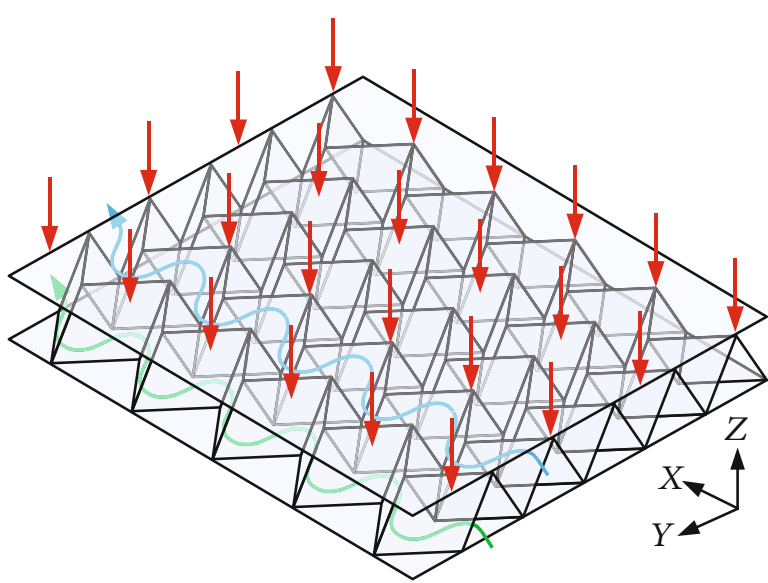

FIgURE 1: Illustration of a heated V-pattern folded core sandwich structure subjected to forced convection.

Typically, a unit cell of V-pattern folded core can be completely described by a set of four independent geometric parameters as shown in Figure 2(a). Using terms in [22], the geometric model is defined by the folded core height $H$, half of the zigzag lines pitch $S$, the saw-tooth lines pitch $2 \mathrm{~L}$, and the zigzag lines amplitude $W$. In reality, when core's web thickness is involved, the folding edge consists more of a plateau than a sharp edge, which reduces the curvature and the extent of potential imperfections. It is assumed that the folded core has uniform web thickness, as shown in Figure 2(b).

2.2. Boundary Conditions and Material Properties. A mass flow rate of $0.0016 \mathrm{~kg} / \mathrm{s}$ with a constant static temperature of $300 \mathrm{~K}$ entering through the streamwise periodic boundary is prescribed for all cases. The direction of the inlet flow is perpendicular to the inlet cross-section. The top-face sheet is subjected to a constant uniform heat flux of $20000 \mathrm{~W} / \mathrm{m}^{2}$ while adiabatic wall is set for the bottom-face sheet.

Aluminium 2024-T851 is used for both folded core and face sheets. Kerosene [23] is chosen as the coolant. Since influences of geometric parameters are mainly investigated in this paper, constant material properties are adopted. Material properties are shown in Table 1.

2.3. Data Reduction Parameters. For fully developed flow and heat transfer in a periodic channel, information obtained from a representative unit cell can be utilized to describe the whole structure. To evaluate the flow and heat transfer characteristics, three nondimensional quantities (the Reynolds number Re, the Darcy friction factor $f$, and the Nusselt number $\mathrm{Nu}$ ) are introduced. Re and $f$ based on the folded core height $H$ are defined as

$$
\begin{gathered}
\operatorname{Re}_{H}=\frac{\rho_{f} u_{0} H}{\mu_{f}}, \\
f_{H}=\left(\frac{\Delta p}{L_{\mathrm{P}}} \cdot H\right) \cdot\left(\frac{1}{\rho_{f} u_{0}^{2} / 2}\right),
\end{gathered}
$$




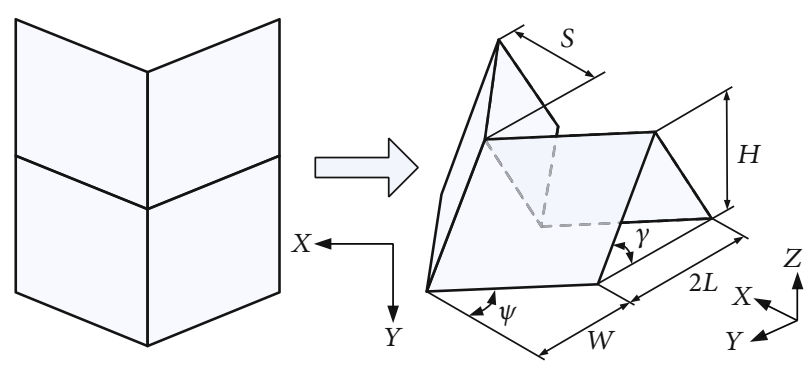

(a)

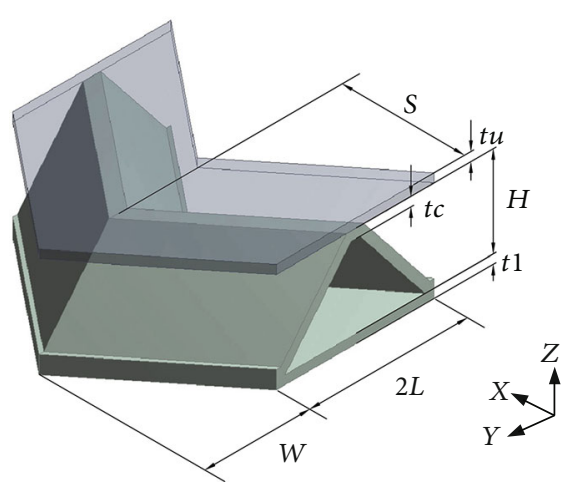

(b)

Figure 2: Geometric parameters of (a) a typical V-pattern folded core unit cell and (b) a unit cell when web thickness is considered.

TABle 1: Material properties.

\begin{tabular}{lcc}
\hline Parameter & $\mathrm{Al} 2024-\mathrm{T} 851$ & Kerosene \\
\hline$\rho\left(\mathrm{kg} \cdot \mathrm{m}^{-3}\right)$ & 2780 & 802.77 \\
$k\left(\mathrm{~W} \cdot \mathrm{m}^{-1} \cdot \mathrm{K}^{-1}\right)$ & 153.94 & 0.1403 \\
$c_{p}\left(\mathrm{~J} \cdot \mathrm{kg}^{-1} \cdot \mathrm{K}^{-1}\right)$ & 852.34 & 1914.6 \\
\hline
\end{tabular}

where $u_{0}$ is the mean inlet velocity, $\rho_{f}$ and $\mu_{f}$ are the density and viscosity of the coolant, respectively, and $\Delta p / L_{P}$ refers to the pressure drop per unit length (i.e., 2S).

At a streamwise location $x$, the perimeter averaged heat transfer coefficient is defined as

$$
\bar{h}_{x}=\frac{\bar{q}_{\mathrm{w}}(x)}{\bar{T}_{\mathrm{w}}(x)-\bar{T}_{f}(x)}
$$

where $\bar{q}_{\mathrm{w}}(x)$ and $\bar{T}_{\mathrm{w}}(x)$ are peripherally averaged wall heat flux and temperature, respectively, and $\bar{T}_{f}(x)$ is the bulk fluid temperature. The overall mean heat transfer coefficient in one unit cell can then be obtained from the spatial average of $\bar{h}_{x}$ as

$$
\bar{h}_{\mathrm{eff}}=\frac{1}{x_{2}-x_{1}} \int_{x_{1}}^{x_{2}} \bar{h}_{x} d x \text {. }
$$

Therefore, the corresponding $\mathrm{Nu}$ based on folded core height $H$ can be defined as

$$
\mathrm{Nu}_{H}=\frac{\bar{h}_{\text {eff }} H}{k_{f}} .
$$

2.4. Computational Grid. Hexahedral grid was used for the whole computational domain, and meshes are refined around the fluid-solid interface to better resolve the boundary layer. The fluid domain and the solid domain were divided by common nodes, which could improve the accu- racy of data transmission at the fluid-solid interface. Convergence studies were conducted to establish an estimated accuracy of the results and to establish mesh independence of the numerical solutions. The influence was examined on the basis of the $f_{H}$ and $\mathrm{Nu}_{H}$ dependence on the element number. The values of $f_{H}$ and $\mathrm{Nu}_{H}$ were taken from the central unit for a typical three-unit cell length $\mathrm{V}$-pattern folded core panel with a uniform velocity and temperature profile at the inlet boundary. The results of the convergence study are presented in Figure 3, and it can be seen that a monotonic convergence toward the final solution is displayed in the figure. In addition, convergence studies were conducted for several other Reynolds numbers and yielded comparable results. The mesh size with 694650 volume elements was eventually settled upon for the model as the resulting solution differed only marginally from a finer mesh. Illustration of a typical computational mesh is shown in Figure 4.

\section{Computational Method}

3.1. Governing Equations. In the present study, some assumptions are considered in the numerical simulation: (1) the flow and heat transfer are steady; (2) the fluid is incompressible and Newtonian; (3) natural convection and radiation heat transfer are neglected; (4) viscous dissipation heat is not considered; (5) thermophysical properties of the material do not change with temperature. Based on above assumptions, the governing equations can be expressed as follows.

Continuity equation

$$
\frac{\partial u_{j}}{\partial x_{j}}=0(j=1,2,3)
$$

where $u_{j}$ is the fluid velocity vector.

Momentum equation

$$
\rho u_{j} \frac{\partial u_{i}}{\partial x_{j}}=-\frac{\partial p}{\partial x_{i}}+\frac{\partial \tau_{i j}}{\partial x_{j}}(i, j=1,2,3),
$$

where $\rho$ is the fluid density, $p$ stands for the pressure, and $\tau_{i j}$ 


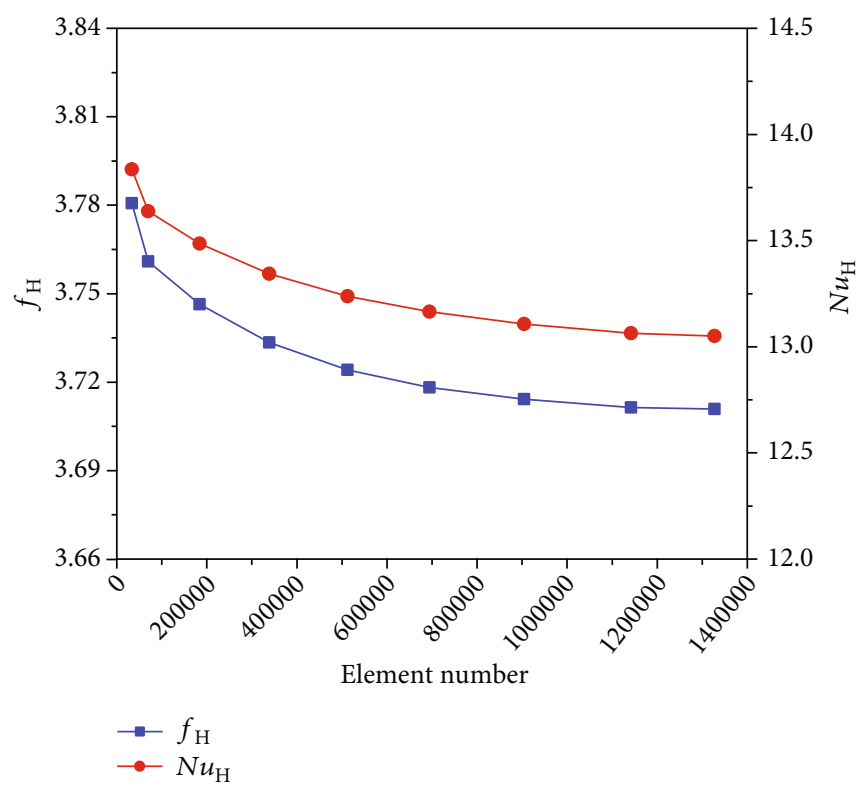

FIgURE 3: Mesh convergence study.

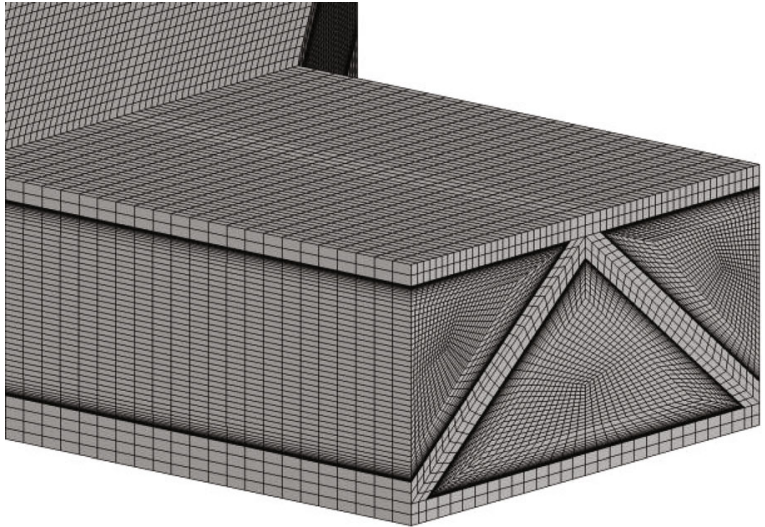

FIgURE 4: Computational grids.

refers to the viscous stress under incompressible condition and can be expressed as

$$
\tau_{i j}=\mu\left(\frac{\partial u_{i}}{\partial x_{j}}+\frac{\partial u_{j}}{\partial x_{i}}\right)(i, j=1,2,3) .
$$

Energy equation

$$
\rho c_{p} u_{j} \frac{\partial T_{\mathrm{f}}}{\partial x_{j}}=\frac{\partial}{\partial x_{j}}\left(k_{\mathrm{f}} \frac{\partial T_{\mathrm{f}}}{\partial x_{j}}\right)(j=1,2,3),
$$

where $T_{f}$ is the fluid temperature and $c_{p}$ and $k_{f}$ stand for the specific heat and thermal conductivity of the fluid, respectively.
For the solid part, the steady heat conduction differential equation can be expressed as

$$
\frac{\partial}{\partial x_{j}}\left(k_{\mathrm{s}} \frac{\partial T_{\mathrm{s}}}{\partial x_{j}}\right)=0(j=1,2,3),
$$

where $T_{\mathrm{s}}$ is the solid temperature and $k_{\mathrm{s}}$ is the thermal conductivity of the solid.

The temperature and heat flux are continuous in the fluid-solid interface and can be expressed as

$$
\left.T_{s}\right|_{w}=\left.T_{f}\right|_{w},
$$

$$
\left.k_{s} \frac{\partial T_{s}}{\partial n}\right|_{w}=\left.k_{f} \frac{\partial T_{f}}{\partial n}\right|_{w} .
$$

3.2. Numerical Procedure. A three-dimensional model is considered to model fully developed, steady-state laminar fluid flow and heat transfer within V-pattern folded core sandwich structures. Due to the periodic characteristic of the V-pattern folded core, flow across the computational unit cell of the folded core sandwich panel is assumed to be fully developed both hydrodynamically and thermally. The assumption is reasonable for a unit cell placed apart from the inlet and outlet region. Thus, both flow and heat transfer calculations in geometries are periodically repetitive in the streamwise direction $X$ and spanwise direction $Y$. Taking a single representative unit cell from the structure and imposing periodic boundary conditions in two directions is desired to minimize the computational expense. A pressure drop occurs across translationally periodic boundaries in the streamwise direction while no pressure drop occurs across the periodic planes in the spanwise direction. This can be summed up to a periodic heat transfer problem 


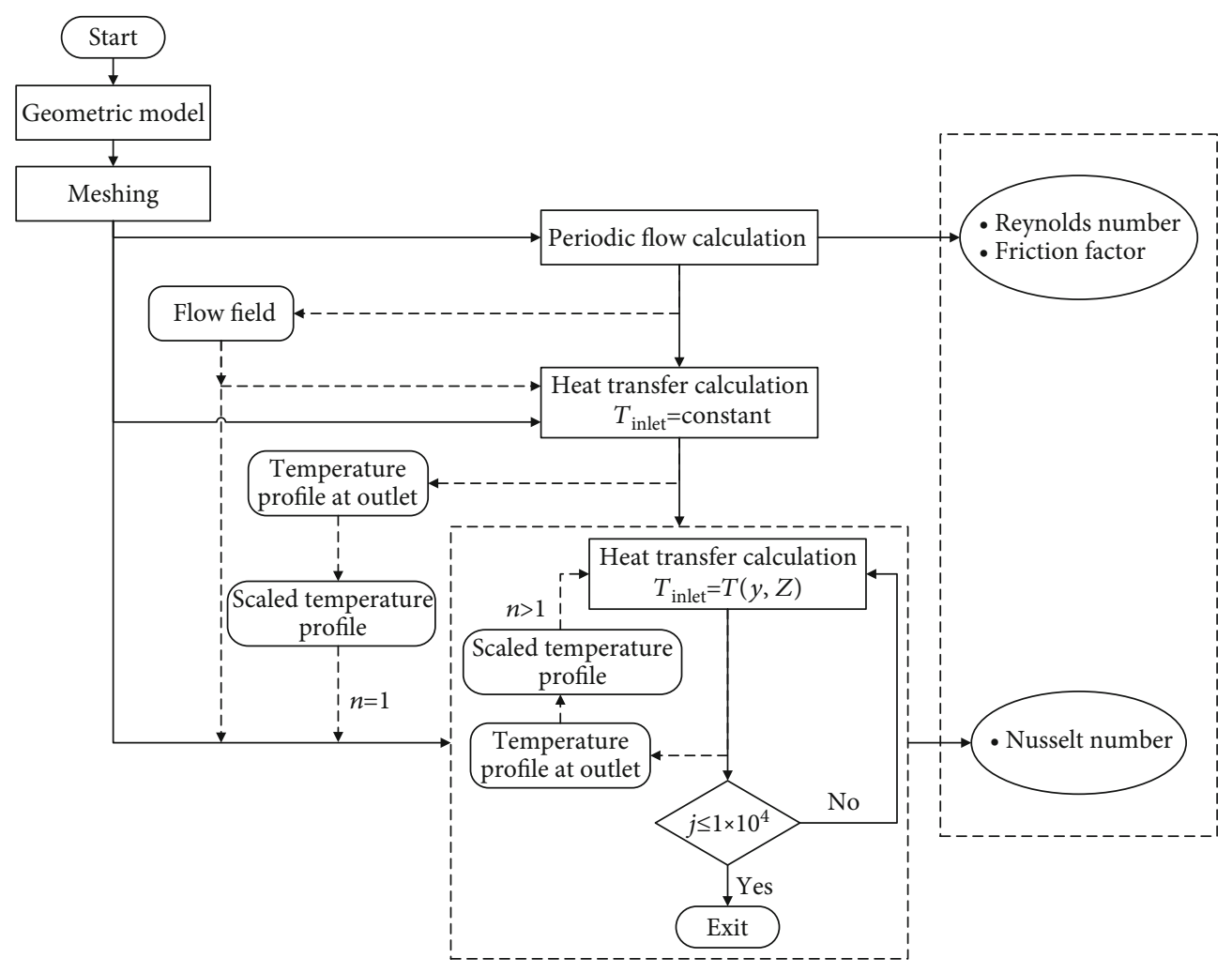

FIgURE 5: Illustration of the analysis procedure.

for a wavy structure with two different types of periodic boundary conditions.

Therefore, a two-step simulation method is developed to achieve a fully developed periodic solution, and details are shown in Figure 5. ANSYS CFX is adopted to perform numerical simulations, where a vertex-based finite volume method is used to solve the Navier-Stokes and energy equations. All simulations are conducted using the high resolution advection scheme. RMS residual below $10^{-6}$ and global imbalance below 0.01 are taken as the convergence criteria.

First, fully developed periodic flow is solved using ANSYS CFX without heat transfer. Two different types of periodic boundary conditions are defined in two directions with a mass flow rate specified in the streamwise direction. Both velocity and pressure field are computed in a single simulation.

Sequentially, the heat transfer is solved leaving the flow field unaltered. To determine the heat transfer characteristics of the structure under thermal fully developed conditions, an iterative procedure is required. The energy transport equation is first solved with a uniform inlet temperature $T_{\text {inlet }}=T_{\text {ref }}=$ constant and the velocity field obtained from the previous step. Afterwards, the outlet temperature field of the first calculation is scaled by the difference between the mass flow averaged outlet temperature and the initial inlet temperature and then reapplied to the inlet for the second iteration. This process is repeated several times until a thermally developed state is achieved. Here, a stopping criteria based on the average Nusselt number $\mathrm{Nu}_{H}$ is given by

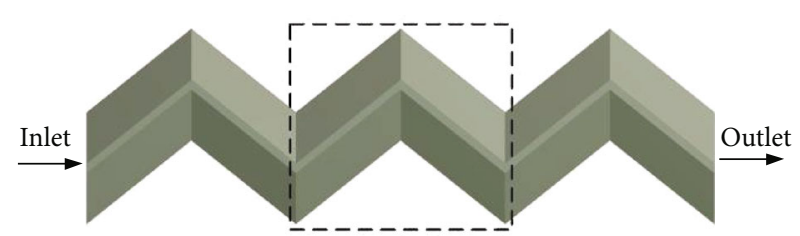

FIgURE 6: Schematic of a three-unit model.

$$
j=\left|\frac{\left(\mathrm{Nu}_{H}\right)_{n+1}-\left(\mathrm{Nu}_{H}\right)_{n}}{\left(\mathrm{Nu}_{H}\right)_{n}}\right| \leq 0.0001
$$

However, the temperature gradient information was not included during iterations [24]. Heat fluxes across the solid periodic interface were also neglected as adiabatic boundary conditions were used. To overcome this, a number of repeating units of geometry need to be included in order to ensure that the heat transfer is fully developed in the unit of interest. Generally, three repeating units are sufficient when all data are extracted from the central unit, as shown in Figure 6. All above procedures are integrated using Isight [25], which makes a good preparation for later analyses and optimization.

For cases of constant wall heat flux, which is mainly considered in this paper, temperature profiles are similar in shape at streamwise locations separated by the period length $2 S$. And the temperature difference between two locations is proportional to the wall heat flux [26]. To gain an initial understanding of the issue, a typical three-unit folded core 


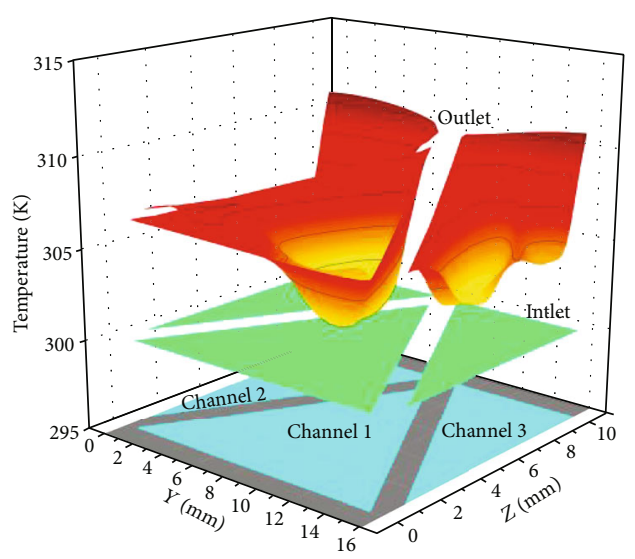

(a)

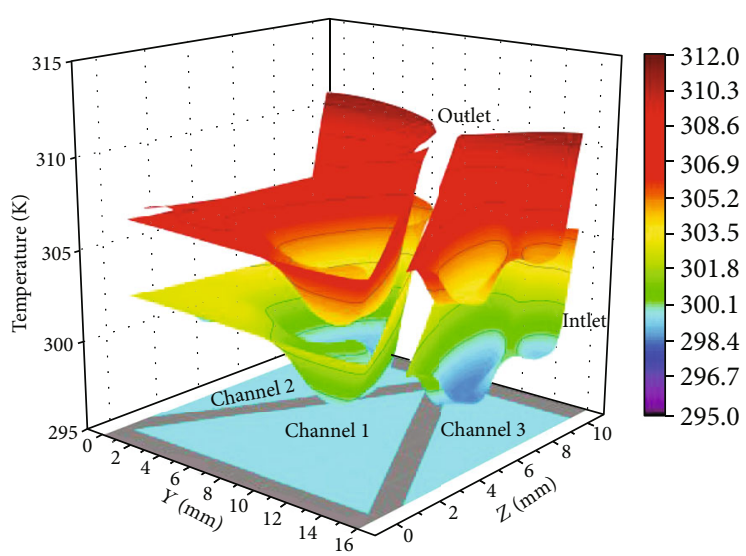

(b)

FIGURE 7: Temperature profiles of the inlet and outlet in (a) the first heat transfer simulation and (b) the fifth iteration.

structure (see Figure 6) is analysed using the previously mentioned two-step method. Temperature profiles of the inlet and outlet for the first heat transfer analysis and iteration five are shown in Figures 7(a) and 7(b), respectively. It is seen that the shapes of the temperature profiles at the entrance and exit are similar after five iterations. It can be considered that the thermally fully developed state has been reached at the 14th cell.

3.3. Method Validation. In order to confirm the accuracy of the method, the Fanning friction factor $\mathrm{C}_{\mathrm{f}}$ and Nusselt number $\mathrm{Nu}$ for laminar fully developed flow and heat transfer in a straight channel with an equilateral triangular crosssection were calculated. Table 2 shows a comparison of $C_{f} \bullet$ Re product and $\mathrm{Nu}$ with values taken from reference [27]. The agreement is greater than $99 \%$ for both parameters.

\section{Results and Discussion}

Folded core sandwich structure has an excellent designability due to its various geometric parameters. The influences of geometric parameters on the flow and heat transfer characteristics are investigated. The folded core sandwich structure affects the coolant flow mainly by the flow channel's tortuosity and cross-section, which can be characterized by the geometric parameters $S, W, H$, and $L$, respectively. Two independent parameters $\psi=\arctan (W / S)$ and $\gamma=\arctan (H / L)$, as shown in Figure 2(a), with a constant folded core unit cell length $(2 S=30 \mathrm{~mm})$ and height $(H=10 \mathrm{~mm})$ are investigated.

Figure 8 shows the flow and heat transfer characteristic variations with the folded core tortuosity along the flow direction. In particular, the wavy channel turns into a straight one when $\psi$ equals to zero. It is seen that both pressure drop and heat transfer performance increase with the angle $\psi$, which is quite obvious since flows in tortuous channels normally give rise to pressure drop penalty and heat transfer enhancement relative to flow in straight channels. It is noted that the heat transfer enhancement is greater than the pressure drop penalty at small turning angles. However, with the continuing increase of the tortuosity, the pressure
TABLE 2: Comparison of flow and heat transfer characteristics.

\begin{tabular}{lccc}
\hline Parameter & $\begin{array}{c}\text { Reference } \\
{[27]}\end{array}$ & $\begin{array}{c}\text { Present } \\
\text { simulation }\end{array}$ & $\begin{array}{c}\text { Percentage difference } \\
(\%)\end{array}$ \\
\hline$C_{f} \cdot \operatorname{Re}$ & 13.333 & 13.352 & 0.142 \\
$\mathrm{Nu}$ & 1.892 & 1.896 & 0.211 \\
\hline
\end{tabular}

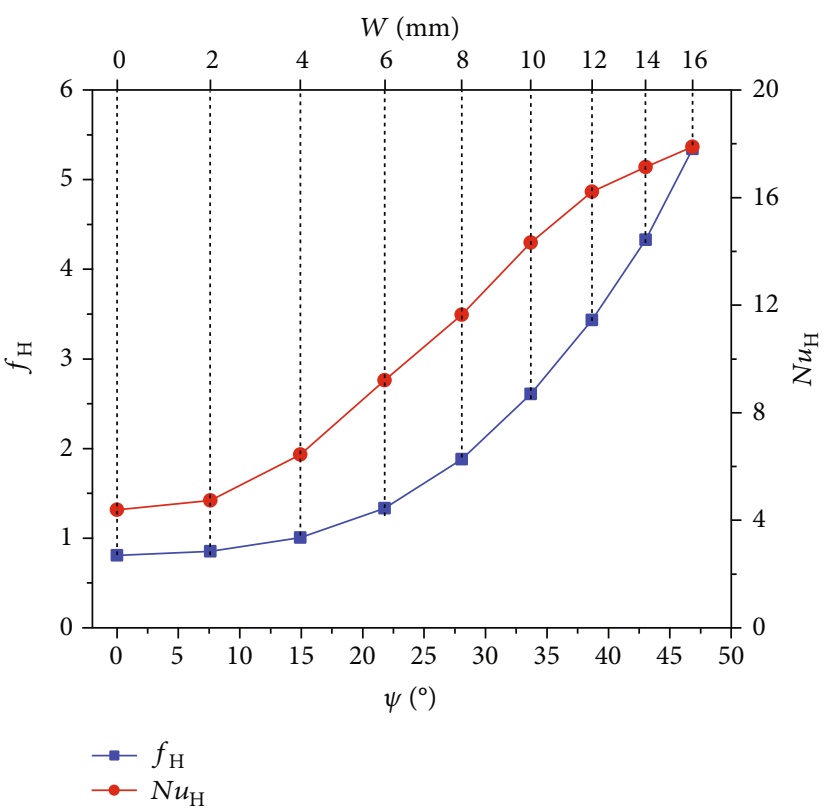

FIGURE 8: Friction factors and Nusselt numbers as a function of angle $\psi$.

drop grows rapidly while the heat transfer enhancement slows down.

Figure 9 shows the influence of the inclined angle $\gamma$ on the flow and heat transfer characteristics. It is seen that both indexes increase with the cross-section changing from an obtuse triangle to an acute triangle. The increase rate of the pressure drop is greater than that of the heat transfer performance before the inclined angle $\gamma$ reaches about $50^{\circ}$. 


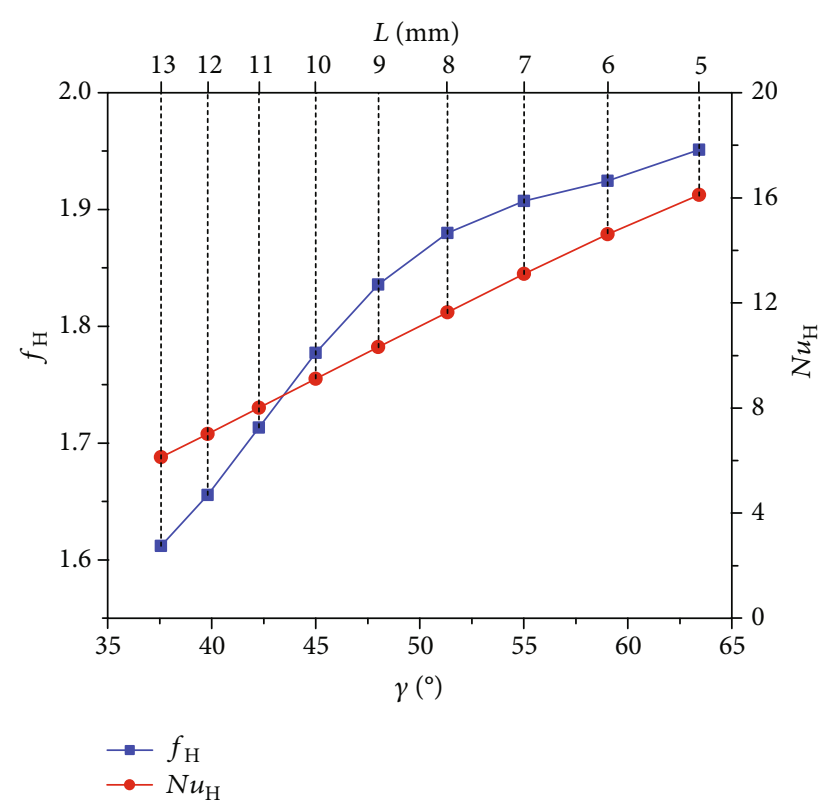

FIGURE 9: Friction factors and Nusselt numbers as a function of angle $\gamma$.

TABLE 3: Ranges of design variables.

\begin{tabular}{lcccc}
\hline Design variables & $H$ & $L$ & $S$ & $W$ \\
\hline Value $(\mathrm{mm})$ & $8-12$ & $6-12$ & $12-20$ & $4-12$ \\
\hline
\end{tabular}

From Figure 8, structures with a small $\gamma$ are more likely to offer efficient heat dissipation. However, it should be noted that constant mass flow rate and folded core height are used in this investigation. Thus, the coolant inlet velocity, as well as the Reynolds number, varies with the cross-sectional area. There might be a different relation among these parameters investigated at a constant Reynolds number, which is not included in the current study.

\section{Optimum Design}

Normally, a structure with good heat transfer behaviour is inclined to suffer a relatively large pressure loss, which makes it necessary to conduct the optimum design for efficient heat dissipation. The heat dissipation performance of the structure can be easily gauged by the ratio of the average Nusselt number $\mathrm{Nu}_{H}$ to the friction factor $f_{H}$ [28]. Consequently, the corresponding objective function of the optimization problem is simply given by

$$
\Pi=\frac{\mathrm{Nu}_{H}}{f_{H}} .
$$

All four geometric parameters are considered as design variables while the folded core and face sheet thicknesses are taken as a fixed value of $1 \mathrm{~mm}$. Based on the previous study of the influence of geometric parameters on the flow and heat transfer performance, a specific restriction which would avoid severely tortuous flow channel or extremely
TABLE 4: Evaluation of RBF approximate models.

\begin{tabular}{lcc}
\hline Parameter & RMSE & $R^{2}$ \\
\hline$f_{H}$ & 0.0039 & 0.9998 \\
$\mathrm{Nu}_{H}$ & 0.0059 & 0.9995 \\
$\Pi$ & 0.0101 & 0.9987 \\
$\bar{\rho}$ & 0.0026 & 0.9999 \\
\hline
\end{tabular}

TABle 5: The optimal geometric dimensions.

\begin{tabular}{lcccc}
\hline Design variables & $H$ & $L$ & $S$ & $W$ \\
\hline Value $(\mathrm{mm})$ & 10.85 & 8.36 & 16.42 & 5.87 \\
\hline
\end{tabular}

TABLE 6: Comparison of predicted results with CFD results.

\begin{tabular}{lccc}
\hline Parameter & RBF & CFD & Percentage difference (\%) \\
\hline$f_{H}$ & 1.2810 & 1.2812 & -0.0161 \\
$\mathrm{Nu}_{H}$ & 8.0430 & 8.0231 & 0.2483 \\
$\Pi$ & 6.2625 & 6.2620 & 0.0080 \\
$\bar{\rho}$ & 0.1500 & 0.1501 & -0.0408 \\
\hline
\end{tabular}

obtuse-triangular and acute-triangular cross-section was imposed when setting the ranges of design variables. The corresponding parameter ranges are shown in Table 3 . The relative density of the folded core is taken as a constraint to prevent the structure from being too heavy; thus, an upper bound of core's relative density $\bar{\rho}$ is specified.

The optimization problem can be succinctly described as:

$$
\left.\begin{array}{l}
\max \Pi \\
\text { s.t. } \bar{\rho} \leq 0.15
\end{array}\right\} \text {. }
$$

First, 200 samples were selected in the design space by using Latin Hypercube Sampling (LHS) technique. These samples were simulated using the previously proposed CFD model and then used to construct approximate models based on the radial basis functions (RBF). 20 random points were used to evaluate the accuracy of the approximate models. Details are shown in Table 4, where RMSE represents root mean square error and $R^{2}$ refers to the coefficient of determination. It is seen that the approximate models are of high precision and can be used to predict the structural performances.

Afterwards, RBF approximate models were invoked to replace the time-consuming CFD models during optimization where sequential quadratic programming was employed for seeking optimal solutions. The optimal geometric dimensions are shown in Table 5. It is seen that $W$ is relatively small to gain a higher $\Pi$, as the pressure loss increases dramatically for a severely tortuous channel. An additional CFD analysis for the optimal parameters was also conducted. The comparison of the RBF predictions and CFD results is shown in Table 6. It is evident that the agreement is excellent, with less than $1 \%$ difference for all interested 


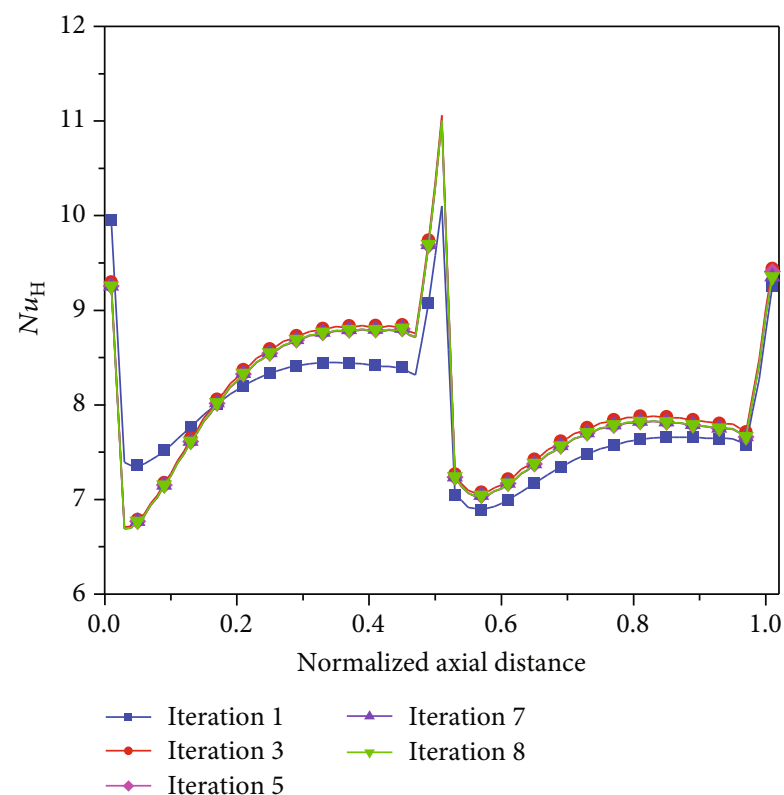

FIGURE 10: Local perimeter averaged Nusselt number in the streamwise direction for iterations $1,3,5,7$, and 8 .

parameters, which indicates that the approximate models have fairly good accuracy.

Specifically, the CFD analysis using the previously mentioned two-step simulation approach shows that eight iterations are needed for the optimal parameters to satisfy the stopping criteria. Figure 10 shows how the perimeter averaged Nusselt number varies through the central unit along the flow direction with increasing iterations. It is seen that the curves were almost coincide with each other after five iterations, which further supports the proposed simulation methodology.

\section{Conclusions}

In this paper, the heat transfer performance and flow state of coolant in a folded core sandwich structure subjected to forced convection conditions have been numerically investigated. A two-step approach to model fully developed laminar flow and heat transfer process is presented. The key conclusions can be drawn as follows:

(1) The proposed two-step simulation method can simulate periodic heat transfer with only a few unit cells, which greatly reduces the amount of calculation in every single run. Simulation of a straight channel with an equilateral triangular cross-section was used to validate the accuracy and feasibility of the methodology

(2) The influences of geometric parameters on the flow and heat transfer characteristics are investigated. Two independent parameters $\psi=\arctan (W / S)$ and $\gamma=\arctan (H / L)$ were introduced, which characterize the tortuosity of the channel and the angle of the cross-sectional triangle, respectively. The results show that with the continuing increase of the tortuosity, the pressure drop grows rapidly while the heat transfer enhancement slows down

(3) Finally, the geometric parameters of folded core, $H$, $L, S$, and $W$, were optimized based on an RBF approximation model with the objective of higher heat transfer efficiency and lower relative density. The optimization results show that a structure with a gently tortuous flow channel dissipates heat more efficient for conditions used in the current study

\section{Nomenclature}

$C_{f}$ : Fanning friction factor

$c_{p}: \quad$ Specific heat capacity, $\mathrm{J} \cdot \mathrm{kg}^{-1} \cdot \mathrm{K}^{-1}$

$f: \quad$ Darcy friction factor

$h$ : $\quad$ Heat transfer coefficient, $\mathrm{W} \cdot \mathrm{m}^{-2} \cdot \mathrm{K}^{-1}$

$H$ : Folded core height, $\mathrm{m}$

$k$ : Thermal conductivity, $\mathrm{W} \cdot \mathrm{m}^{-1} \cdot \mathrm{K}^{-1}$

$p$ : $\quad$ Pressure, $\mathrm{Pa}$

$q$ : $\quad$ Heat flux, $\mathrm{W} \cdot \mathrm{m}^{-2}$

Re: Reynolds number

$T: \quad$ Temperature, $\mathrm{K}$

$u$ : Velocity, $\mathrm{m} \cdot \mathrm{s}^{-1}$

$u$ : $\quad$ Velocity vector, $\mathrm{m} \cdot \mathrm{s}^{-1}$

$\mu$ : Dynamic viscosity, $\mathrm{N} \cdot \mathrm{s} \cdot \mathrm{m}^{-2}$

$\rho: \quad$ Density, $\mathrm{kg} \cdot \mathrm{m}^{-3}$

$\tau: \quad$ Viscous stress, $\mathrm{Pa}$

$\psi, \gamma$ : Angle, degree.

\section{Data Availability}

The data used to support the findings of this study are included within the article.

\section{Conflicts of Interest}

The authors declare that there is no conflict of interest regarding the publication of this paper.

\section{Acknowledgments}

This work was supported by the Funding of Jiangsu Innovation Program for Graduate Education (grant no. CXLX13 163), the Fundamental Research Funds for the Central Universities (grant no. NZ2016101), and a project funded by the Priority Academic Program Development of Jiangsu Higher Education Institutions (PAPD).

\section{References}

[1] S. Heimbs, "Virtual testing of sandwich core structures using dynamic finite element simulations," Computational Materials Science, vol. 45, no. 2, pp. 205-216, 2009.

[2] Z. Chen, Z. J. Wang, and J. Y. Zhi, "Thermal-mechanical analysis of actively cooled folded core sandwich panels," Journal of Solid Rocket Technology, vol. 37, no. 4, pp. 545-550, 2014. 
[3] S. Fischer, "Aluminium foldcores for sandwich structure application: mechanical properties and FE-simulation," ThinWalled Structures, vol. 90, pp. 31-41, 2015.

[4] Z. Wang, V. I. Khaliulin, and E. Skripkin, "Geometry design method of folded structure," Journal of Nanjing University of Aeronautics \& Astronautics, vol. 34, no. 1, pp. 6-11, 2002.

[5] B. P. Abbott, R. Abbott, T. D. Abbott et al., "Observation of gravitational waves from a binary black hole merger," Physical Review Letters, vol. 116, no. 6, article 061102, pp. 363-377, 2016.

[6] S. Heimbs, P. Middendorf, S. Kilchert, A. F. Johnson, and M. Maier, "Experimental and numerical analysis of composite folded sandwich core structures under compression," Applied Composite Materials, 2007.

[7] S. Heimbs, J. Cichosz, M. Klaus, S. Kilchert, and A. F. Johnson, "Sandwich structures with textile-reinforced composite foldcores under impact loads," Composite Structures, vol. 92, no. 6, pp. 1485-1497, 2010.

[8] T. Wen, J. Tian, T. J. Lu, D. T. Queheillalt, and H. N. G. Wadley, "Forced convection in metallic honeycomb structures," International Journal of Heat and Mass Transfer, vol. 49, no. 19-20, pp. 3313-3324, 2006.

[9] T. Wen, F. Xu, and T. J. Lu, "Structural optimization of twodimensional cellular metals cooled by forced convection," International Journal of Heat and Mass Transfer, vol. 50, no. 13-14, pp. 2590-2604, 2007.

[10] T. Kim, H. P. Hodson, and T. J. Lu, "Contribution of vortex structures and flow separation to local and overall pressure and heat transfer characteristics in an ultralightweight lattice material," International Journal of Heat and Mass Transfer, vol. 48, no. 19-20, pp. 4243-4264, 2005.

[11] L. Gao and Y. G. Sun, "Fluid flow and heat transfer characteristics of composite lattice core sandwich structures," Journal of Thermophysics and Heat Transfer, vol. 28, no. 2, pp. 258-269, 2014.

[12] L. Gao, Y. Sun, and L. Cong, "Effect of inclination angle on active cooling performance of composite sandwich structure with lattice truss cores," Acta Materiae Compositae Sinica, vol. 29, no. 5, pp. 47-54, 2013.

[13] J. Rakow and A. M. Waas, "Response of actively cooled metal foam sandwich panels exposed to thermal loading," AIAA Journal, vol. 45, no. 2, pp. 329-336, 2007.

[14] S. S. Feng, J. J. Kuang, T. J. Lu, and K. Ichimiya, "Heat transfer and pressure drop characteristics of finned metal foam heat sinks under uniform impinging flow," Journal of Electronic Packaging, vol. 137, no. 2, pp. 021014.1-021014.12, 2015.

[15] A. Shenoy, M. Sheremet, and I. Pop, Convective Flow and Heat Transfer from Wavy Surfaces: Viscous Fluids, Porous Media, and Nanofluids, CRC Press, 2016.

[16] M. M. Rashidi, S. Ghahremanian, D. Toghraie, and P. Roy, "Effect of solid surface structure on the condensation flow of argon in rough nanochannels with different roughness geometries using molecular dynamics simulation," International Communications in Heat and Mass Transfer, vol. 117, 2020.

[17] M. M. Rashidi, M. Sadri, and M. A. Sheremet, "Numerical simulation of hybrid nanofluid mixed convection in a lid-driven square cavity with magnetic field using high-order compact scheme," Nanomaterials, vol. 11, no. 9, p. 2250, 2021.
[18] M. Khoshvaght-Aliabadi, A. Zamzamian, and F. Hormozi, "Wavy channel and different nanofluids effects on performance of plate-fin heat exchangers," Journal of Thermophysics and Heat Transfer, vol. 28, no. 3, pp. 474-484, 2014.

[19] M. Khoshvaght-Aliabadi and F. Rad Hormozi, " $\mathrm{Al}_{2} \mathrm{O}_{3}$-water nanofluid inside wavy mini-channel with different cross-sections," Journal of the Taiwan Institute of Chemical Engineers, vol. 58, pp. 8-18, 2016.

[20] M. Khoshvaght-Aliabadi, S. M. Hassani, and H. MazloumiS, "Enhancement of laminar forced convection cooling in wavy heat sink with rectangular ribs and $\mathrm{Al}_{2} \mathrm{O}_{3}$ /water nanofluids," Experimental Thermal and Fluid Science, vol. 89, pp. 199210, 2017.

[21] M. Khoshvaght-Aliabadi, S. Deldar, A. Salimi, and M. M. Rashidi, "Effects of cross-section geometry on performance of corrugated miniature heat sink: uniform, convergent, divergent, and hybrid cases," International Communications in Heat and Mass Transfer, vol. 127, 2021.

[22] V. I. Khaliulin and I. V. Dvoeglazov, "On technological problems of fabrication of relief designs by isometric transformation of thin sheet," Transactions of Nanjing University of Aeronautics \& Astronautics, vol. 18, no. 1, 2001.

[23] F. Zhong, X. Fan, G. Yu, J. Li, and C. J. Sung, "Heat transfer of aviation kerosene at supercritical conditions," Journal of Thermophysics and Heat Transfer, vol. 23, no. 3, pp. 543-550, 2009.

[24] N. R. Rosaguti, D. F. Fletcher, and B. S. Haynes, "Laminar flow and heat transfer in a periodic serpentine channel with semicircular cross-section," International Journal of Heat and Mass Transfer, vol. 49, no. 17-18, pp. 2912-2923, 2006.

[25] "Isight 5.9 user's guide," Dassault Systemes Simulia Crop, 2014.

[26] S. V. Patankar, C. H. Liu, and E. M. Sparrow, "Fully developed flow and heat transfer in ducts having streamwise-periodic variations of cross-sectional area," Journal of Heat Transfer, vol. 99, no. 2, p. 180, 1977.

[27] R. K. Shah, "Laminar flow friction and forced convection heat transfer in ducts of arbitrary geometry," International Journal of Heat and Mass Transfer, vol. 18, no. 7-8, pp. 849-862, 1975.

[28] J. Tian, T. Kim, T. J. Lu et al., "The effects of topology upon fluid-flow and heat-transfer within cellular copper structures," International Journal of Heat and Mass Transfer, vol. 47, no. 14-16, pp. 3171-3186, 2004. 